\title{
Albuminuria rather than glomerular filtration rate is associated with vascular endothelial function in patients with type 2 diabetes
}

Yoshinori Kakutani, Tomoaki Morioka, Katsuhito Mori, Yuko Yamazaki, Akinobu Ochi, Masafumi Kurajoh, Shinya Fukumoto, Atsushi Shioi, Tetsuo Shoji, Masaaki Inaba, Masanori Emoto

\begin{tabular}{|c|c|}
\hline Citation & Journal of Diabetes and its Complications. 34(12); 107702. \\
\hline Issue Date & 2020-12 \\
\hline Type & Journal Article \\
\hline Textversion & Author \\
\hline Highlights & $\begin{array}{l}\text { - The association between ACR or eGFR and FMD was investigated in type } 2 \text { diabetes. } \\
\text { - FMD was impaired in advanced stages of CKD based on eGFR and ACR categories. } \\
\text {-ACR, but not eGFR, was independently associated with FMD. } \\
\text { - Albuminuria would be a surrogate for endothelial dysfunction in type } 2 \text { diabetes. }\end{array}$ \\
\hline Rights & $\begin{array}{l}\text { (C) } 2020 \text { Elsevier Inc. This manuscript version is made available under the } \\
\text { CC-BY-NC-ND } 4.0 \text { License. http://creativecommons.org/licenses/by-nc-nd/4.0/. } \\
\text { This is the accepted manuscript version. The article has been published in final form } \\
\text { at https://doi.org/10.1016/j.jdiacomp.2020.107702. }\end{array}$ \\
\hline DOI & 10.1016/j.jdiacomp.2020.107702 \\
\hline
\end{tabular}

\author{
Self-Archiving by Author(s) \\ Placed on: Osaka City University Repository
}

Yoshinori Kakutani, Tomoaki Morioka, Katsuhito Mori, Yuko Yamazaki, Akinobu Ochi, Masafumi Kurajoh, Shinya Fukumoto, Atsushi Shioi, Tetsuo Shoji, Masaaki Inaba, Masanori Emoto. (2020). Albuminuria rather than glomerular filtration rate is associated with vascular endothelial function in patients with type 2 diabetes. Journal of Diabetes and its Complications. 34, 107702.

Doi:10.1016/j.jdiacomp.2020.107702 
Original Research Article

\section{Albuminuria rather than glomerular filtration rate is associated with vascular endothelial function in patients with type 2 diabetes}

Yoshinori Kakutani ${ }^{\text {a }}$, Tomoaki Morioka ${ }^{\text {a* }}$, Katsuhito Mori ${ }^{\text {b, }}$ Yuko Yamazaki ${ }^{\text {a }}$, Akinobu Ochi ${ }^{\text {a }}$, Masafumi Kurajoh $^{\text {a }}$, Shinya Fukumoto ${ }^{c}$, Atsushi Shioi d,e, Tetsuo Shoji d,e , Masaaki Inaba ${ }^{\text {a,e }}$, Masanori Emoto ${ }^{a}$

${ }^{a}$ Department of Metabolism, Endocrinology and Molecular Medicine, Osaka City University Graduate School of Medicine. 1-4-3, Asahi-machi, Abeno-ku, Osaka, 545-8585 Japan

b Department of Nephrology, Osaka City University Graduate School of Medicine. 1-4-3, Asahi-machi, Abeno-ku, Osaka, 545-8585 Japan

${ }^{c}$ Department of Premier Preventive Medicine, Osaka City University Graduate School of Medicine. 1-4-3, Asahi-machi, Abeno-ku, Osaka, 545-8585 Japan

${ }^{\mathrm{d}}$ Department of Vascular Medicine, Osaka City University Graduate School of Medicine. 1-4-3, Asahi-machi, Abeno-ku, Osaka, 545-8585 Japan

e Vascular Science Center for Translational Research, Osaka City University Graduate School of Medicine. 1-4-3, Asahi-machi, Abeno-ku, Osaka, 545-8585 Japan

* Corresponding author: Tomoaki Morioka, M.D, Ph.D.

Department of Metabolism, Endocrinology and Molecular Medicine, Osaka City University

Graduate School of Medicine. 1-4-3, Asahi-machi, Abeno-ku, Osaka, 545-8585 Japan.

Tel.: +81-6-6645-3806; fax: +81-6-6645-3808.

E-mail: m-tomo@med.osaka-cu.ac.jp

Word count: abstract, 199; main text, 3572; tables, 884; total 4456 excluding references. Two figures and three tables are included in the manuscript.

\section{Conflict of interest}

The authors declare that they have no competing interests related to the present study. 


\section{Abstract}

Aims: Albuminuria and reduced glomerular filtration rate (GFR) are manifestations of diabetic kidney disease and are both shown to be associated with cardiovascular outcomes. However, the differential association of albuminuria and reduced GFR with endothelial dysfunction, an early feature of atherosclerotic vascular damage, remains unclear. In this study, we investigated the association between albuminuria or estimated GFR (eGFR) and flow-mediated dilatation (FMD), a marker of endothelial function, in patients with type 2 diabetes.

Methods: This study included 633 patients with type 2 diabetes. The FMD of the brachial artery was measured by ultrasonography. Albuminuria was evaluated by urinary albumin-to-creatinine ratio (ACR).

Results: The mean FMD and eGFR, and the median value of ACR were 6.7\%, 66.5 $\mathrm{mL} / \mathrm{min} / 1.73 \mathrm{~m}^{2}$ and $12.5 \mathrm{mg} / \mathrm{g}$ creatinine, respectively. Impaired FMD was found in patients with advanced stages of chronic kidney disease based on both GFR and albuminuria categories. Multivariate analysis after adjusting for potential confounders revealed that ACR, but not eGFR, was significantly and inversely associated with FMD.

Conclusions: Albuminuria is associated with FMD, independently of traditional cardiovascular risk factors in patients with type 2 diabetes. This study suggests a close relationship between albuminuria, rather than reduced GFR, and endothelial dysfunction in type 2 diabetes.

Keywords: Albuminuria, Glomerular filtration rate, Endothelial function, Flowmediated dilatation, Type 2 diabetes 


\section{Introduction}

The prevalence of diabetic kidney disease (DKD), a common complication of diabetes, has been increased due primarily to the increase in type 2 diabetes throughout the world ${ }^{1,2}$. DKD, defined as diabetes with increased albuminuria, decreased glomerular filtration rate (GFR), or both, is not only a leading cause of end-stage renal disease, but also is strongly associated with cardiovascular disease (CVD) ${ }^{2,3}$. Accumulating evidence indicates that increased albuminuria and decreased estimated GFR (eGFR) are independently and additively associated with the increased risk of CVD events, CVD mortality, and all-cause mortality in type 2 diabetes patients ${ }^{4-7}$ as well as in the general population ${ }^{3,8}$.

The flow-mediated dilatation (FMD) of the brachial artery is a non-invasive measure of vascular endothelial function ${ }^{9,10}$ which are shown to be impaired in individuals with risk factors for CVD, reflecting the early stage of atherosclerotic vascular damage ${ }^{11}$. Moreover, the predictive value of FMD for CVD outcomes has been shown by a number of studies in the general population ${ }^{12}$, patients with CVD ${ }^{11,13}$, and those with type 2 diabetes ${ }^{14}$. Regarding the impact of DKD on endothelial function, a number of studies have recently examined the relationship between albuminuria and/or eGFR and FMD in patients with diabetes ${ }^{15-25}$. These studies consistently demonstrated a trend for reduced FMD in patients with advanced CKD stages by albuminuria ${ }^{15}$, 17, 18, 20, 22, 23, 25 and GFR 15-17, 19, 21 categories. However, limited studies have explored the independent association of albuminuria or eGFR with FMD by multivariate analysis with adjustment for potential confounders including eGFR, albuminuria, or both in type 2 diabetes patients ${ }^{15,17,23,25}$.

Those prior reports have provided inconsistent results regarding the association of albuminuria or eGFR with FMD in type 2 diabetes patients 15, 17, 23, 25. The independent determinants of FMD identified in those studies were both albuminuria and eGFR ${ }^{17}$, only 
albuminuria ${ }^{23,25}$, or neither of them ${ }^{15}$. These prior studies vary in terms of study population, number of participants, degree of kidney disease, and/or methods of evaluating albuminuria. Thus far, it remains unclear which of increased albuminuria, reduced GFR, or both affect vascular endothelial dysfunction, a surrogate marker of vascular risk, in type 2 diabetes patients. The present study aimed to investigate the differential association of eGFR and albuminuria with FMD in a large number of Japanese patients with type 2 diabetes.

\section{Subjects, Materials and Methods}

\subsection{Study design and subjects}

In this cross-sectional study, we consecutively enrolled 633 patients with type 2 diabetes who were admitted to the Diabetes Center of the Osaka City University Hospital for the purpose of glycemic control, education, and/or evaluation of diabetic complications between 2009 and 2016. A diagnosis of type 2 diabetes was made on the basis of the criteria of American Diabetes Association ${ }^{26}$ and Japan Diabetes Society ${ }^{27}$. Type 1 diabetes patients were excluded from the present study. Complication of CVDs, including coronary artery disease, cerebrovascular diseases, or peripheral artery disease was confirmed by medical records. A smoker was defined as a current smoker or an ex-smoker. The subjects were divided into GFR categories and albuminuria categories of chronic kidney disease (CKD) ${ }^{28}$. This study was performed in accordance with the Declaration of Helsinki (1975, as revised in 2013) and the Ethical Guidelines for Medical and Health Research Involving Human Subjects (The Japanese Ministry of Health, Labour and Welfare, 2014). The study protocol was approved by the ethics committee of the institution (No. 307). We obtained written informed consent from all subjects.

\subsection{Physical and laboratory measurements}


Blood pressure was determined by the conventional cuff method with an automatic sphygmomanometer after subjects had rested for at least $15 \mathrm{~min}$. Blood tests were performed after an overnight fast. Biochemical parameters were analyzed by a standard laboratory method at the Central Clinical Laboratory of the Osaka City University Hospital ${ }^{29-31}$. Glycated hemoglobin A1c (HbA1c) levels were estimated as National Glycohemoglobin Standardization Program equivalent values (\%) using the converting formula established by the Japan Diabetes Society ${ }^{27}$. eGFR was calculated using the formula by the Japanese Society of Nephrology: eGFR $\left(\mathrm{mL} / \mathrm{min} / 1.73 \mathrm{~m}^{2}\right)=194 \times$ serum creatinine ${ }^{-1.094} \times \mathrm{Age}^{-0.287}(\times 0.739 \text { if female })^{32}$. Albuminuria was determined by urinary albumin-to-creatinine ratio $(\mathrm{mg} / \mathrm{g})$ in a spot urine sample.

\subsection{Measurements of FMD and NMD by ultrasound system}

We measured FMD of the brachial artery using an ultrasound system (UNEXEF; Unex Co. Ltd., Nagoya, Japan), as described in our previous reports ${ }^{19,29,30}$. Following FMD measurement, endothelium-independent, nitroglycerin-mediated dilatation (NMD) was also measured for 473 subjects without contraindications for nitroglycerin, such as bradycardia, hypotension, or glaucoma, as previously described ${ }^{19,29,30}$. Briefly, after a 15-min interval for vessel recovery, a single sublingual dose of glyceryl trinitrate $(75 \mu \mathrm{g})$ was administered, and the maximum dilatation of the brachial artery at the same point was measured during at least 1 min after the initiation of maximum dilatation. Measurements were performed in a quiet, air-conditioned room at $25.0^{\circ} \mathrm{C}$ for inpatients who had not taken any foods, caffeine, tobacco, or exercise for at least 12 hours before examinations, according to the International Brachial Artery Reactivity Task Force guideline ${ }^{9}$.

\subsection{Statistics}


Data are expressed as the number $(\%)$, mean \pm standard deviation, or median (interquartile range). For comparison among eGFR or ACR categories of CKD, $\chi^{2}$-test or ANOVA with posthoc Dunn's test was performed as appropriate. Correlation between variables was analyzed by Pearson correlation coefficient. ACR was converted into logarithmic scale before being used for regression analysis. To explore the association of eGFR or log [ACR] with FMD or NMD, multiple regression analyses were performed with adjustment for age, sex (male $=1$, female $=0)$, duration of diabetes, body mass index (BMI), systolic blood pressure, HbA1c level, low-density lipoprotein (LDL) cholesterol level, smoking (yes=1, no=0), complication of CVDs (yes=1, no=0), treatment with statins $(\mathrm{yes}=1, \mathrm{no}=0$ ), treatment with renin-angiotensin-aldosterone system (RAAS) inhibitors (yes $=1$, no $=0$ ), and treatment with antihyperglycemic agents $($ yes $=1$, no $=0$ ) including insulin, sulfonylureas, biguanides, and dipeptidyl peptidase (DPP)-4 inhibitors. A pvalue of $<0.05$ was considered statistically significant. Statistical analyses were performed using the JMP ${ }^{\circledR} 13$ software (SAS Institute Inc., Cary, NC, USA).

\section{Results}

\subsection{Clinical Characteristics of the participants}

The clinical characteristics of the total population are shown in Table 1. The mean age, BMI, $\mathrm{HbA1c}$, and the median duration of diabetes in the total population were 62 years, $26.0 \mathrm{~kg} / \mathrm{m}^{2}$, $8.6 \%$, and 10 years, respectively. Twenty percent of the subjects had history of CVDs, including coronary artery disease, cerebrovascular disease, and/or peripheral artery disease. Prevalence of subjects receiving statin and RAAS inhibitors were $45.1 \%$, and $40.8 \%$, respectively. Regarding the antihyperglycemic agents, 67 subjects $(11.0 \%)$ were treated with dietary therapy alone, 200 (32.3\%) with sulfonylureas, 185 (30.0\%) with biguanides, 215 (35.0\%) with DPP-4 inhibitors, 64 $(10.4 \%)$ with thiazolidinediones, 14 (2.3\%) with glucagon-like peptide-1 receptor agonists, and 
$221(38.2 \%)$ with insulin therapy. The mean eGFR and the median urinary ACR of the total population were $66.5 \mathrm{~mL} / \mathrm{min} / 1.73 \mathrm{~m}^{2}$, and $12.5 \mathrm{mg} / \mathrm{g}$, respectively.

Clinical characteristics of subjects in each CKD stage based on eGFR and ACR categories were also shown in Table 2. The number (proportion) of subjects categorized into G1/2 stage $\left(\mathrm{eGFR} \geq 60 \mathrm{~mL} / \mathrm{min} / 1.73 \mathrm{~m}^{2}\right)$, G3a stage $\left(\mathrm{eGFR} 45-59 \mathrm{~mL} / \mathrm{min} / 1.73 \mathrm{~m}^{2}\right)$, G3b stage $(\mathrm{eGFR} 30-$ $\left.44 \mathrm{~mL} / \mathrm{min} / 1.73 \mathrm{~m}^{2}\right)$, and $\mathrm{G} 4 / 5$ stage $\left(\mathrm{eGFR}<30 \mathrm{~mL} / \mathrm{min} / 1.73 \mathrm{~m}^{2}\right)$ were $392(61.9 \%), 118(18.6 \%)$, 63 (10.0\%), and 60 (9.5\%), respectively. Patients in G3a, G3b and G4/5 stages were older and had longer duration of diabetes than those in G1/2 stage. Patients in G4/5 stage had lower HbA1c and HDL-cholesterol than those in G1/2 stage. The number (proportion) of subjects categorized into A1stage (ACR < $30 \mathrm{mg} / \mathrm{g}$ ), A2 stage (ACR $30-299 \mathrm{mg} / \mathrm{g}$ ), and A3 stage (ACR $\geq 300 \mathrm{mg} / \mathrm{g}$ ) were $425(67.1 \%), 101(16.0 \%)$, and 107 (16.9\%), respectively. No significant difference in age or HbA1c was observed between the stages. Patients in the advanced albuminuria stages showed longer duration of diabetes, higher systolic blood pressure, higher frequency of RAAS inhibitor users, and higher prevalence of CVDs than those in A1 stage.

\subsection{FMD and NMD of the participants}

The mean values of FMD and NMD in the total population were $6.7 \%$ and $14.3 \%$, respectively (Table 1). Comparisons of FMD and NMD among CKD stages based on eGFR or ACR category are shown in Figure 1. Patients in G3a and G4/5 stages showed significantly lower FMD (mean, $5.5 \%$ and $5.0 \%$, respectively) than those in G1/2 stage (7.3\%) (Fig. 1A). Patients in A3 stage showed significantly lower FMD (mean, 5.4\%) than those in A1 stage (7.1\%) (Fig. 1B). Patients in G4/5 stage showed significantly lower NMD (mean, 9.9\%) than those in G1/2 stage (15.3\%) (Fig. 1C). Patients in A2 and A3 stages showed lower NMD (mean, 11.6\% and $13.1 \%$, respectively) than those in A1 stage (15.2\%) (Fig. 1D). 


\subsection{Association between eGFR or ACR and FMD/NMD}

Correlation analysis was conducted to examine the relationship between CKD and vascular dysfunction. Both eGFR (Fig. 2A) and log [ACR] (Fig. 2B) were significantly correlated with FMD in the univariate analysis. Significant correlation with NMD was also found in both eGFR (Fig. 2C) and $\log [\mathrm{ACR}]$ (Fig. 2D).

To determine the independent association of eGFR and ACR with FMD, multiple regression analysis was performed with adjustment for potential confounders including age, sex, duration of diabetes, BMI, systolic blood pressure, HbA1c, LDL-cholesterol, smoking, complication of CVDs, use of statins, use of RAAS inhibitors, and use of antihyperglycemic agents including insulin, sulfonylureas, biguanides, and DPP-4 inhibitors (Table 3). In performing multiple regression analysis, missing value of the adjustment factors was examined. A total of 94 (14.8\%) subjects were found to have some missing values, and the parameters with missing values were as follows; duration of diabetes $(n=12)$, BMI $(n=11)$, systolic blood pressure $(n=19)$, HbA1c $(\mathrm{n}=1)$, LDL-cholesterol $(\mathrm{n}=14)$, smoking $(\mathrm{n}=1)$, complication of CVDs $(\mathrm{n}=3), \operatorname{ACR}(\mathrm{n}=2)$, use of statins ( $n=15)$, use of RAAS inhibitors $(n=16)$, use of insulin $(n=16)$, use of sulfonylureas $(n=11)$, use of biguanides ( $n=11)$, and use of DPP-4 inhibitors $(n=11)$.

Besides the traditional risk factors for CVDs, including age, male sex, and complication of CVDs, $\log [\mathrm{ACR}]$ was found to be an independent determinant of FMD (model 2). Notably, the association between $\log [\mathrm{ACR}]$ and FMD remained significant even after additional adjustment for eGFR (model 3). In contrast, eGFR showed no independent association with FMD (models 1 and 3). On the other hand, neither eGFR nor log [ACR] was found to be an independent determinant of NMD. 


\section{Discussion}

The present study examined the differential association of eGFR and albuminuria with FMD in patients with type 2 diabetes and demonstrated that ACR, but not eGFR, was significantly associated with FMD. This study highlights three important findings. First, the association between ACR and FMD was independent of eGFR and other traditional risk factors for CVDs. Second, eGFR was not an independent determinant of FMD. Third, neither albuminuria nor eGFR was associated with NMD. Our data provide additional evidence on the close relationship between albuminuria and vascular endothelial dysfunction, an early feature of vascular damage, in type 2 diabetes patients.

This study revealed a close association between albuminuria and FMD in a large number of subjects with type 2 diabetes and a wide range of renal function. In agreement with prior studies 15, 17, 18, 20, 22, 23, 25, impaired FMD was observed in patients with increased albuminuria. Of importance, our study identified a close correlation between ACR and impaired FMD, which was independent of potential confounders including age, BMI, systolic blood pressure, HbA1c, LDLcholesterol, smoking habit, and eGFR. Several studies evaluated the cross-sectional relationship between albuminuria and the ultrasonically measured FMD of the brachial artery $15,17,23,25$. In accordance with our results, the presence of albuminuria was shown to be significantly associated with impaired FMD, after adjusting for other covariates in a population-based study of elderly Caucasians including 260 subjects with type 2 diabetes ${ }^{23}$. Other studies in Japanese patients with type 2 diabetes $\left(\mathrm{n}=158^{25}\right.$ and $\left.480{ }^{17}\right)$ also identified ACR ${ }^{25}$ or the presence of albuminuria ${ }^{17}$ as a determinant of FMD independent of traditional CVD risk factors. On the other hand, a small study in Chinese type 2 diabetes patients $(n=58){ }^{15}$ failed to demonstrate a significant association between urinary albumin excretion and FMD. It should be noted, however, that the subjects with diabetic nephropathy in that study had much severe renal insufficiency (mean GFR, 22.6 
$\left.\mathrm{mL} / \mathrm{min} / 1.72 \mathrm{~m}^{2}, \mathrm{n}=26\right){ }^{15}$ compared to those in our study and others ${ }^{17,23,25}$. In the present study, we found a linear correlation between FMD and ACR as a continuous variable, instead of categorical variable of the presence or absence albuminuria ${ }^{17,23}$. In addition, this study enrolled a larger number of diabetic subjects $(n=633)$ who had poorer glycemic control (mean HbA1c, $8.9 \%$ ) than the other prior studies ${ }^{17,23,25}$. Thus, our study would provide additional evidence of the clinical relationship between albuminuria and endothelial dysfunction in type 2 diabetes.

The close relationship between albuminuria and endothelial dysfunction has been evidenced by previous studies not only in type 2 diabetes patients $15,17,18,20,22,23,25$, but also in non-diabetic subjects ${ }^{33}, 34$. Moreover, several studies have demonstrated that biochemical estimates of endothelial dysfunction, such as von Willebrand factor ${ }^{35-37}$, tissue plasminogen activator ${ }^{35}$, and soluble vascular cell adhesion molecule-1 ${ }^{38}$, preceded and predicted the development or progression of albuminuria in both diabetic ${ }^{36,37}$ and non-diabetic ${ }^{35,38}$ individuals, suggesting a causal role of endothelial dysfunction on the development of albuminuria regardless of diabetes status. Although the molecular mechanism remains to be identified, common factors such as chronic, low-grade inflammation and insulin resistance are suggested to underlie the link between endothelial dysfunction and albuminuria ${ }^{39}$. In another view of our data, FMD was shown to be an independent determinant of ACR $(\beta=-0.094, p=0.008)$ along with age, systolic blood pressure, eGFR, and HbA1c (model $R^{2}=0.419, p<0.001$ ). Considering that the majority $(67.1 \%)$ of our study participants were categorized into A1 stage with normoalbuminuria, a linear correlation between ACR and impaired FMD which was independent of the common risk factors suggests that endothelial dysfunction is implicated in albuminuria from the early stages of diabetic nephropathy. Nevertheless, a prospective study would be required to determine the causal relationship between endothelial dysfunction and albuminuria in our study population.

This study failed to demonstrate a significant association between eGFR and FMD. Previous 
studies conducted in type 2 diabetes patients are largely consistent with our results, indicating no significant association between eGFR ${ }^{15,25}$ or serum creatinine level ${ }^{19,23}$ and FMD. Unlike our study and others, a couple of studies have shown a significant association between eGFR and FMD, regardless of the degree of albuminuria, in type 2 diabetes patients ${ }^{16,17}$. Those studies, however, would not contradict our results because one study ${ }^{17}$ evaluated albuminuria by the presence or absence of stage A1 or A2, but not by ACR as a continuous variable. Another study demonstrated a close relationship between eGFR, but not ACR, and FMD, but the number of patients with albuminuria was much smaller (12\%), and the median ACR (4.4 mg/g) was lower than those in our study (33\% and $12.5 \mathrm{mg} / \mathrm{g}$, respectively) ${ }^{16}$. It is worth considering that reduced eGFR was shown to be an independent determinant of endothelial dysfunction in studies of nondiabetic population without albuminuria ${ }^{40,41}$. Taken together, it is suggested that the association between eGFR and FMD depends on the degree of albuminuria in the subjects and that albuminuria, rather than reduced GFR, is related to endothelial dysfunction in patients showing albuminuria

Our study additionally explored the association of eGFR and ACR with NMD, which is not only used as a control test for FMD to differentiate endothelium-dependent from endotheliumindependent vasodilatation, but also is reported to be impaired in subjects with cardiovascular risk factors ${ }^{42}$ and predictive of future CVD events ${ }^{43,44}$. In agreement with prior studies ${ }^{15,18,19}$, we found the impaired NMD in type 2 diabetes patients with advanced diabetic nephropathy. However, neither ACR nor eGFR, but age, BMI, and systolic blood pressure, was revealed to be an independent determinant of NMD in our study. Our results would not rule out the possible involvement of CKD in the impaired NMD, because various factors related to $\mathrm{CKD}$, including abnormal bone metabolism, oxidative stress, chronic, low-grade inflammation, and uremic toxins, are suggested to cause smooth muscle cell dysfunction in CKD ${ }^{45}$. Indeed, several studies showed 
albuminuria as an independent determinant of NMD in type 2 diabetes patients with normal kidney function (mean eGFR greater than $90 \mathrm{~mL} / \mathrm{min} / 1.73 \mathrm{~m}^{2}$ ) ${ }^{18,46}$ and in non-diabetic and hypertensive patients ${ }^{33,34}$. An independent association between eGFR and NMD was also shown in type 2 diabetes patients with very low levels of albuminuria ${ }^{16}$. A study which included diabetic patients with severe renal insufficiency (mean eGFR, $22.6 \mathrm{~mL} / \mathrm{min} / 1.72 \mathrm{~m}^{2}$ ) showed that urinary albumin excretion was independently associated with NMD ${ }^{15}$. Taken together, it is suggested that the association of albuminuria and eGFR with NMD depends on the degree of albuminuria and/or eGFR in the subjects. Mildly reduced eGFR (mean, $66.5 \mathrm{~mL} / \mathrm{min} / 1.73 \mathrm{~m}^{2}$ ) and the low level of ACR (median, $12.5 \mathrm{mg} / \mathrm{g}$ ) of the participants may have caused the absence of significant association between ACR or eGFR and NMD in our study.

The present study has several limitations. First, as discussed above, this was a cross-sectional study which could not establish a causal relationship between eGFR and/or ACR and endothelial dysfunction. Second, this study lacks non-diabetic controls, with which a close relationship between endothelial dysfunction and albuminuria could be confirmed regardless of diabetes status. Third, many of the participants in this study had poor glycemic control, which is recently suggested to be a major factor in the overestimation of GFR in diabetic patients ${ }^{47}$. Fourth, we had a certain number of missing values for the parameters included in the multiple regression analysis, that could distort our results. Lastly, the coefficient of determination in the multivariate analysis was quite low (model $R^{2}=0.113$ ), as is the case of a previous large-scale population study (model $\left.R^{2}=0.160\right)^{48}$. Therefore, factors other than ACR, major CVD risk factors, and medications could still remain to be identified as significant determinants of FMD. The strength of this study was that we enrolled the largest number of type 2 diabetes patients ( $n=633)$ among other related studies 15-20, 22-25 which evaluated FMD by ultrasonography. Another strength of the study was that we were able to exclude the possible involvement of medications in the association between ACR 
and FMD by adjusting for use of statins, RAAS inhibitors, and major antihyperglycemic agents in the multivariate analysis.

\section{Conclusions}

This study demonstrated that albuminuria, but not eGFR, is associated with FMD in type 2 diabetes patients, independently of traditional risk factors for CVDs. This study clearly indicated the differential association of albuminuria and eGFR with FMD in a large number of diabetic subjects. Our data suggest a close link between albuminuria, rather than decreased GFR, and endothelial dysfunction, an early marker of vascular damage, in type 2 diabetes. Further longitudinal studies are warranted to determine whether the changes in albuminuria are directly related to the changes in endothelial function in type 2 diabetes patients.

\section{Acknowledgements}

The authors acknowledge the valuable assistance of all the data entry staff at the Department of Metabolism, Endocrinology, and Molecular Medicine, Osaka City University Graduate School of Medicine.

\section{Funding}

This study was partly supported by a Grant-in-Aid for Scientific Research (No. 20591068) from the Japan Society for the Promotion of Science (to Masanori Emoto and Katsuhito Mori). The funding source had no involvement in study design; in the collection, analysis and interpretation of data; in the writing of the report; or in the decision to submit the article for publication. 


\section{Author contributions}

Yoshironi Kakutani, Tomoaki Morioka, Katsuhito Mori, and Masanori Emoto conceived the study, participated in its design and coordination, and helped in the drafting of the manuscript. Yoshinori Kakutani, Tomoaki Morioka and Katsuhito Mori performed the statistical analyses. Yoshinori Kakutani, Yuko Yamazaki enrolled the patients and performed vascular examinations. Akinobu Ochi, Masafumi Kurajoh, Shinya Fukumoto, Atsushi Shioi, Tetsuo Shoji, and Masaaki Inaba contributed to the discussion section and were involved in drafting and revising the manuscript. All authors have read and approved the final version of the manuscript.

\section{Abbreviations}

GFR, glomerular filtration rate; eGFR, estimated GFR; FMD, flow-mediated dilatation; ACR, albumin-to-creatinine ratio; DKD, diabetic kidney disease; CVD, cardiovascular disease; CKD, chronic kidney disease; HbA1c, glycated hemoglobin A1c; NMD, nitroglycerin-mediated dilatation; BMI, body mass index; LDL, low-density lipoprotein; RAAS, renin-angiotensinaldosterone system; DPP, dipeptidyl peptidase. 


\section{References}

1. de Boer IH, Rue TC, Hall YN, Heagerty PJ, Weiss NS, Himmelfarb J. Temporal trends in the prevalence of diabetic kidney disease in the United States. JAMA. 2011;305(24): 25322539. 10.1001/jama.2011.861

2. Tuttle KR, Bakris GL, Bilous RW, et al. Diabetic kidney disease: a report from an ADA Consensus Conference. Am J Kidney Dis. 2014;64(4): 510-533.

10.1053/j.ajkd.2014.08.001

3. Fox CS, Matsushita K, Woodward M, et al. Associations of kidney disease measures with mortality and end-stage renal disease in individuals with and without diabetes: a metaanalysis. Lancet. 2012;380(9854): 1662-1673. 10.1016/S0140-6736(12)61350-6

4. Afkarian M, Sachs MC, Kestenbaum B, et al. Kidney disease and increased mortality risk in type 2 diabetes. $J$ Am Soc Nephrol. 2013;24(2): 302-308. 10.1681/ASN.2012070718 5. Drury PL, Ting R, Zannino D, et al. Estimated glomerular filtration rate and albuminuria are independent predictors of cardiovascular events and death in type 2 diabetes mellitus: the Fenofibrate Intervention and Event Lowering in Diabetes (FIELD) study. Diabetologia. 2011;54(1): 32-43. 10.1007/s00125-010-1854-1

6. Miyake H, Kanazawa I, Sugimoto T. Albuminuria Increases All-Cause Mortality in Japanese Patients with Type 2 Diabetes Mellitus. J Clin Med. 2018;7(9). 10.3390/jcm7090234 7. Wada T, Haneda M, Furuichi K, et al. Clinical impact of albuminuria and glomerular filtration rate on renal and cardiovascular events, and all-cause mortality in Japanese patients with type 2 diabetes. Clin Exp Nephrol. 2014;18(4): 613-620. 10.1007/s10157-013-0879-4 8. Hallan SI, Matsushita K, Sang Y, et al. Age and association of kidney measures with mortality and end-stage renal disease. JAMA. 2012;308(22): 2349-2360. 
10.1001/jama.2012.16817

9. Corretti MC, Anderson TJ, Benjamin EJ, et al. Guidelines for the ultrasound assessment of endothelial-dependent flow-mediated vasodilation of the brachial artery: a report of the International Brachial Artery Reactivity Task Force. J Am Coll Cardiol. 2002;39(2): 257265.

10. Inoue $\mathrm{T}$, Matsuoka $\mathrm{H}$, Higashi $\mathrm{Y}$, et al. Flow-mediated vasodilation as a diagnostic modality for vascular failure. Hypertens Res. 2008;31(12): 2105-2113. 10.1291/hypres.31.2105 JST.JSTAGE/hypres/31.2105 [pii]

11. Tomiyama H, Yamashina A. Non-invasive vascular function tests: their pathophysiological background and clinical application. Circ J. 2010;74(1): 24-33. JST.JSTAGE/circj/CJ-09-0534 [pii]

12. Yeboah J, Folsom AR, Burke GL, et al. Predictive value of brachial flow-mediated dilation for incident cardiovascular events in a population-based study: the multi-ethnic study of atherosclerosis. Circulation. 2009;120(6): 502-509. 10.1161/CIRCULATIONAHA.109.864801 13. Kitta Y, Obata JE, Nakamura T, et al. Persistent impairment of endothelial vasomotor function has a negative impact on outcome in patients with coronary artery disease. J Am Coll Cardiol. 2009;53(4): 323-330. 10.1016/j.jacc.2008.08.074

14. van Sloten TT, Henry RM, Dekker JM, et al. Endothelial dysfunction plays a key role in increasing cardiovascular risk in type 2 diabetes: the Hoorn study. Hypertension. 2014;64(6): 1299-1305. 10.1161/HYPERTENSIONAHA.114.04221

15. Chan WB, Chan NN, Lai CW, et al. Vascular defect beyond the endothelium in type II diabetic patients with overt nephropathy and moderate renal insufficiency. Kidney Int. 2006;70(4): 711-716. 10.1038/sj.ki.5001652

16. Hamilton SJ, Chew GT, Davis TM, Watts GF. Prevalence and predictors of abnormal 
arterial function in statin-treated type 2 diabetes mellitus patients. Metabolism. 2012;61(3): 349357. 10.1016/j.metabol.2011.07.010

17. Ito H, Nakashima M, Meguro K, et al. Flow Mediated Dilatation Is Reduced with the Progressive Stages of Glomerular Filtration Rate and Albuminuria in Type 2 Diabetic Patients without Coronary Heart Disease. J Diabetes Res. 2015;2015: 728127. 10.1155/2015/728127 18. Jawa A, Nachimuthu S, Pendergrass M, Asnani S, Fonseca V. Impaired vascular reactivity in African-American patients with type 2 diabetes mellitus and microalbuminuria or proteinuria despite angiotensin-converting enzyme inhibitor therapy. J Clin Endocrinol Metab. 2006;91(1): 31-35. 10.1210/jc.2005-1632

19. Kawano N, Emoto M, Mori K, et al. Association of endothelial and vascular smooth muscle dysfunction with cardiovascular risk factors, vascular complications, and subclinical carotid atherosclerosis in type 2 diabetic patients. J Atheroscler Thromb. 2012;19(3): 276-284. 20. Makino H, Doi K, Hiuge A, et al. Impaired flow-mediated vasodilatation and insulin resistance in type 2 diabetic patients with albuminuria. Diabetes Res Clin Pract. 2008;79(1): 177-182. 10.1016/j.diabres.2007.08.014

21. Miyagi T, Kohagura K, Ishiki T, et al. Interrelationship between brachial artery function and renal small artery sclerosis in chronic kidney disease. Hypertens Res. 2014;37(9): 863-869. 10.1038/hr.2014.60

22. Papaioannou GI, Seip RL, Grey NJ, et al. Brachial artery reactivity in asymptomatic patients with type 2 diabetes mellitus and microalbuminuria (from the Detection of Ischemia in Asymptomatic Diabetics-brachial artery reactivity study). Am J Cardiol. 2004;94(3): 294-299. 10.1016/j.amjcard.2004.04.022

23. Stehouwer CD, Henry RM, Dekker JM, Nijpels G, Heine RJ, Bouter LM.

Microalbuminuria is associated with impaired brachial artery, flow-mediated vasodilation in 
elderly individuals without and with diabetes: further evidence for a link between microalbuminuria and endothelial dysfunction--the Hoorn Study. Kidney Int Suppl. 2004(92): S42-44. 10.1111/j.1523-1755.2004.09211.x

24. Suetsugu M, Takebayashi K, Aso Y. Association between diabetic microangiopathy and vascular endothelial function evaluated by flow-mediated vasodilatation in patients with type 2 diabetes. Int J Clin Pract. 2007;61(6): 920-926. 10.1111/j.1742-1241.2006.01223.x 25. Yokoyama H, Sone H, Saito K, Yamada D, Honjo J, Haneda M. Flow-mediated dilation is associated with microalbuminuria independent of cardiovascular risk factors in type 2 diabetes - interrelations with arterial thickness and stiffness. J Atheroscler Thromb. 2011;18(9): $744-752$

26. American Diabetes Association. 2. Classification and Diagnosis of Diabetes: Standards of Medical Care in Diabetes-2018. Diabetes Care. 2018;41(Suppl 1): S13-S27. $10.2337 / \mathrm{dc} 18-\mathrm{S} 002$

27. Committee of the Japan Diabetes Society on the Diagnostic Criteria of Diabetes Mellitus, Seino Y, Nanjo K, et al. Report of the committee on the classification and diagnostic criteria of diabetes mellitus. J Diabetes Investig. 2010;1(5): 212-228. 10.1111/j.20401124.2010.00074.x

28. Chapter 1: Definition and classification of CKD. Kidney Int Suppl (2011). 2013;3(1): 19-62. 10.1038/kisup.2012.64

29. Morioka T, Emoto M, Imamura S, et al. Plasma polyunsaturated fatty acid profile is associated with vascular endothelial function in patients with type 2 diabetes. Diab Vasc Dis Res. 2018;15(4): 352-355. 10.1177/1479164118774314

30. Morioka T, Emoto M, Yamazaki Y, et al. Leptin is associated with vascular endothelial function in overweight patients with type 2 diabetes. Cardiovasc Diabetol. 2014;13: 10. 
$10.1186 / 1475-2840-13-10$

31. Morioka T, Emoto M, Yamazaki Y, et al. Plasma soluble leptin receptor levels are associated with pancreatic beta-cell dysfunction in patients with type 2 diabetes. J Diabetes Investig. 2018;9(1): 55-62. 10.1111/jdi.12657

32. Matsuo S, Imai E, Horio M, et al. Revised equations for estimated GFR from serum creatinine in Japan. Am J Kidney Dis. 2009;53(6): 982-992. 10.1053/j.ajkd.2008.12.034

33. Malik AR, Sultan S, Turner ST, Kullo IJ. Urinary albumin excretion is associated with impaired flow- and nitroglycerin-mediated brachial artery dilatation in hypertensive adults. $J$ Hum Hypertens. 2007;21(3): 231-238. 10.1038/sj.jhh.1002143

34. Yun L, Xu R, Zhang L, et al. The role of microalbuminuria in arterial endothelial dysfunction in hypertensive patients with carotid plaques. Int Heart J. 2014;55(2): 153-159. 35. Clausen P, Feldt-Rasmussen B, Jensen G, Jensen JS. Endothelial haemostatic factors are associated with progression of urinary albumin excretion in clinically healthy subjects: a 4year prospective study. Clin Sci (Lond). 1999;97(1): 37-43.

36. Stehouwer CD, Fischer HR, van Kuijk AW, Polak BC, Donker AJ. Endothelial dysfunction precedes development of microalbuminuria in IDDM. Diabetes. 1995;44(5): 561564. 10.2337/diab.44.5.561

37. Stehouwer CD, Nauta JJ, Zeldenrust GC, Hackeng WH, Donker AJ, den Ottolander GJ. Urinary albumin excretion, cardiovascular disease, and endothelial dysfunction in noninsulin-dependent diabetes mellitus. Lancet. 1992;340(8815): 319-323. 10.1016/01406736(92)91401-s

38. Jager A, van Hinsbergh VW, Kostense PJ, et al. C-reactive protein and soluble vascular cell adhesion molecule-1 are associated with elevated urinary albumin excretion but do not explain its link with cardiovascular risk. Arterioscler Thromb Vasc Biol. 2002;22(4): $593-$ 
598. 10.1161/01.atv.0000013786.80104.d4

39. Stehouwer CD. Endothelial dysfunction in diabetic nephropathy: state of the art and potential significance for non-diabetic renal disease. Nephrol Dial Transplant. 2004;19(4): 778781. 10.1093/ndt/gfh015

40. Chong KC, Owens CD, Park M, et al. Relationship between kidney disease and endothelial function in peripheral artery disease. J Vasc Surg. 2014;60(6): 1605-1611. 10.1016/j.jvs.2014.08.105

41. Stam F, van Guldener C, Becker A, et al. Endothelial dysfunction contributes to renal function-associated cardiovascular mortality in a population with mild renal insufficiency: the Hoorn study. J Am Soc Nephrol. 2006;17(2): 537-545. 10.1681/ASN.2005080834

42. Maruhashi T, Soga J, Fujimura N, et al. Nitroglycerine-induced vasodilation for assessment of vascular function: a comparison with flow-mediated vasodilation. Arterioscler Thromb Vasc Biol. 2013;33(6): 1401-1408. 10.1161/ATVBAHA.112.300934

43. Akamatsu D, Sato A, Goto H, et al. Nitroglycerin-mediated vasodilatation of the brachial artery may predict long-term cardiovascular events irrespective of the presence of atherosclerotic disease. J Atheroscler Thromb. 2010;17(12): 1266-1274. 10.5551/jat.5181

44. Kajikawa M, Maruhashi T, Hida E, et al. Combination of Flow-Mediated Vasodilation and Nitroglycerine-Induced Vasodilation Is More Effective for Prediction of Cardiovascular Events. Hypertension. 2016;67(5): 1045-1052. 10.1161/HYPERTENSIONAHA.115.06839

45. Henaut L, Mary A, Chillon JM, Kamel S, Massy ZA. The Impact of Uremic Toxins on Vascular Smooth Muscle Cell Function. Toxins (Basel). 2018;10(6). 10.3390/toxins10060218 46. Furuta M, Ueyama M, Morita S, Yamana A, Sanke T. Combined examination of glyceryl trinitrate-mediated vascular dilation with flow-mediated vascular dilation is essential for assessment of vascular function in type 2 diabetes. J Diabetes Investig. 2013;4(3): 304-309. 
$10.1111 /$ jdi.12021

47. Tsuda A, Ishimura E, Ohno Y, et al. Poor glycemic control is a major factor in the overestimation of glomerular filtration rate in diabetic patients. Diabetes Care. 2014;37(3): 596603. $10.2337 / \mathrm{dc} 13-1899$

48. Benjamin EJ, Larson MG, Keyes MJ, et al. Clinical correlates and heritability of flowmediated dilation in the community: the Framingham Heart Study. Circulation. 2004;109(5): 613-619. 10.1161/01.CIR.0000112565.60887.1E

\section{Figure legends}

Fig. 1. Comparison of FMD $(\mathbf{A}, \mathbf{B})$ and $\operatorname{NMD}(\mathbf{C}, \mathbf{D})$ between the eGFR $(\mathbf{A}, \mathbf{C})$ or ACR $(\mathbf{B}, \mathbf{D})$ categories of CKD in type 2 diabetes patients. Data are presented as mean \pm standard deviation. $P$ values were determined by ANOVA. ${ }^{*}, p<0.05,{ }^{* *}, p<0.01$ vs. G1/2 or A1 by post-hoc Dunn's test. FMD, flow-mediated dilatation; NMD, nitroglycerin-mediated dilatation; eGFR, estimated glomerular filtration rate; ACR, albumin-to-creatinine ratio.

Fig. 2. Correlation of FMD $(\mathbf{A}, \mathbf{B})$ or $\operatorname{NMD}(\mathbf{C}, \mathbf{D})$ with eGFR $(\mathbf{A}, \mathbf{C})$ and ACR $(\mathbf{B}, \mathbf{D})$ in type 2 diabetes patients. ACR was logarithmically transformed. $r$, correlation coefficient by simple regression analysis. FMD, flow-mediated dilatation; NMD, nitroglycerin-mediated dilatation; eGFR, estimated glomerular filtration rate; ACR, albumin-to-creatinine ratio. 
Fig. 1.

A

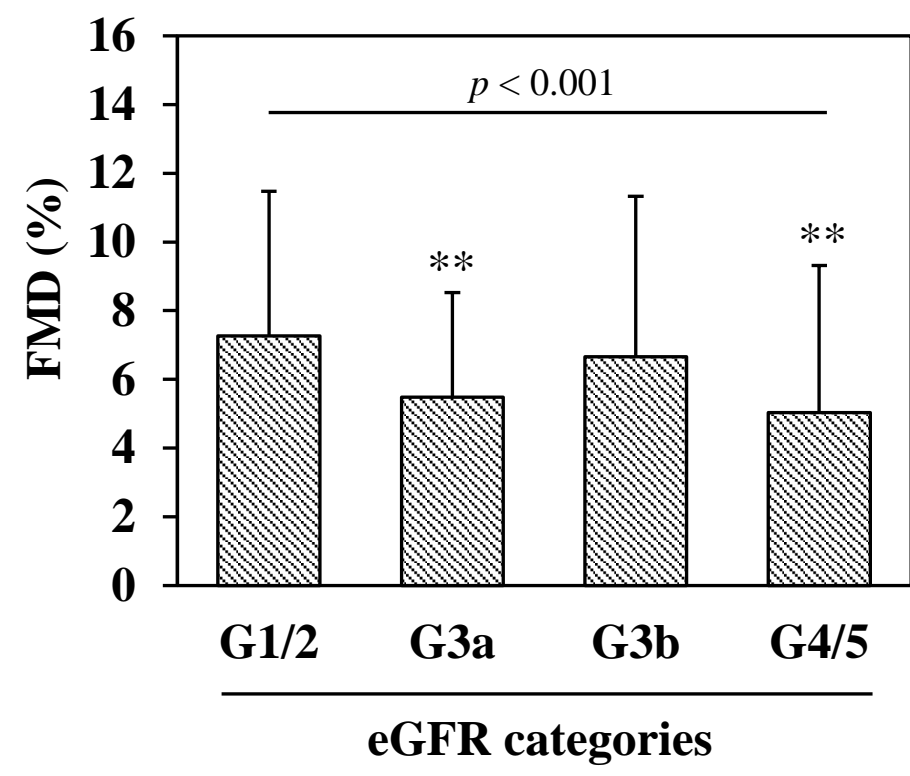

B

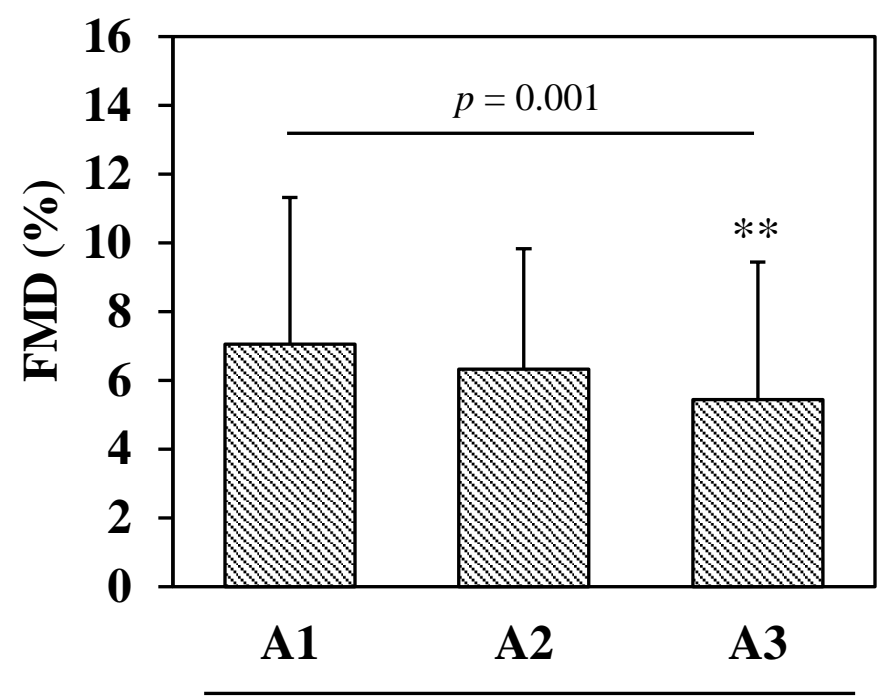

ACR categories

D

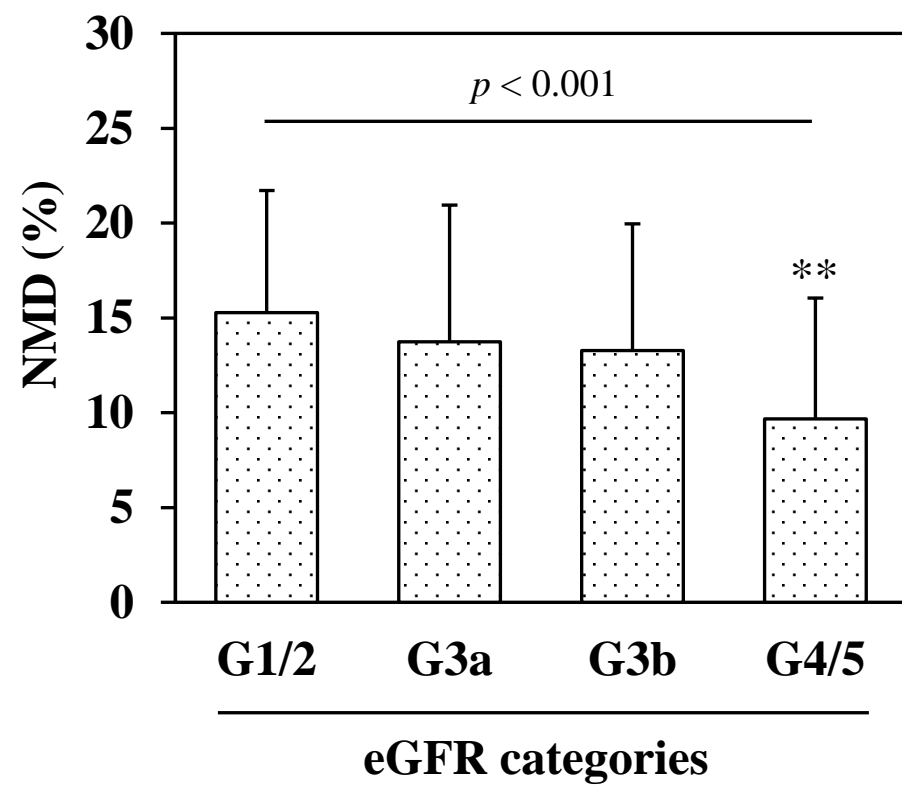

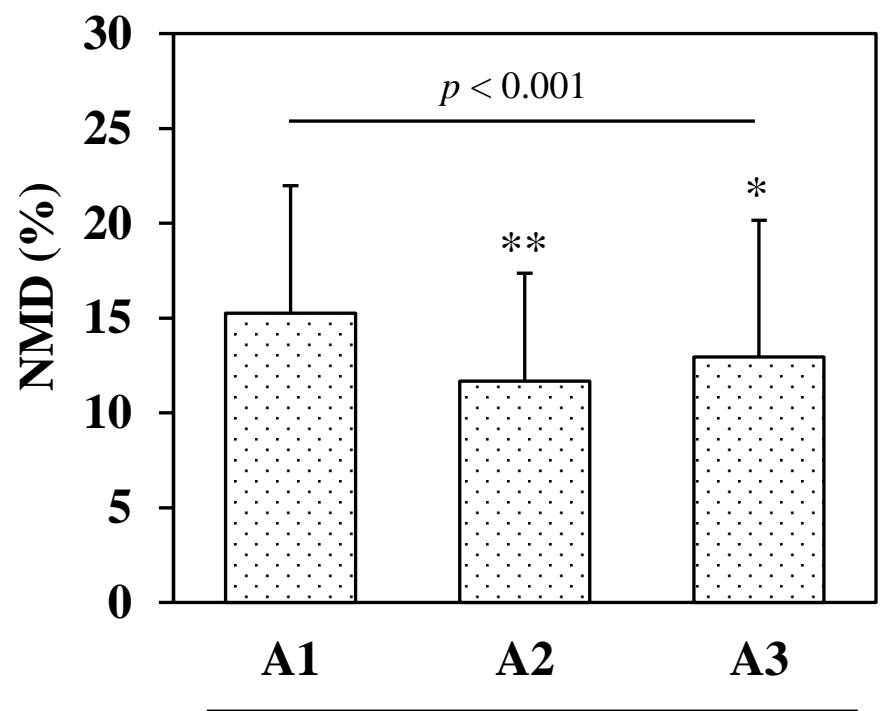

ACR categories 
Fig. 2.

A

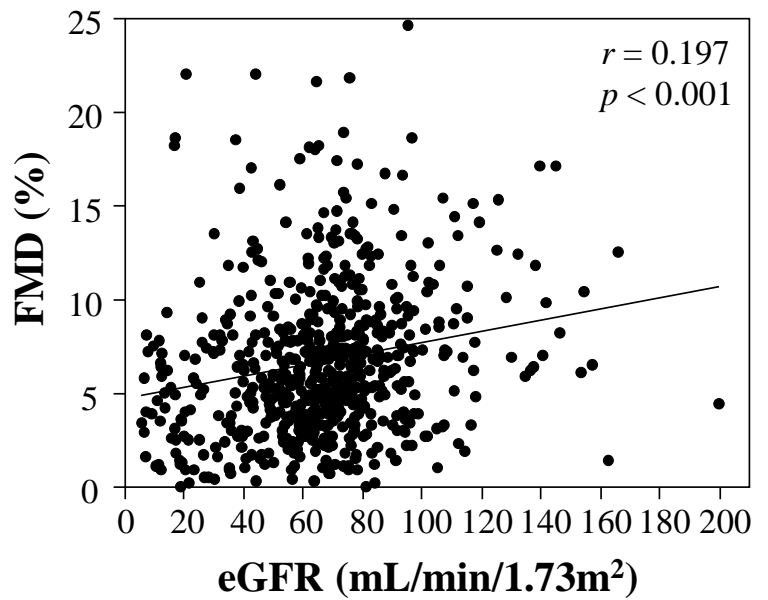

C

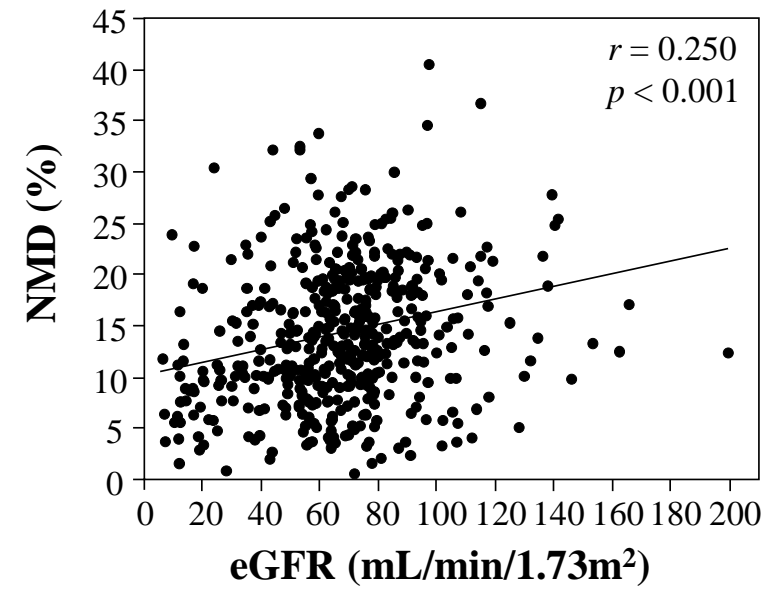

B

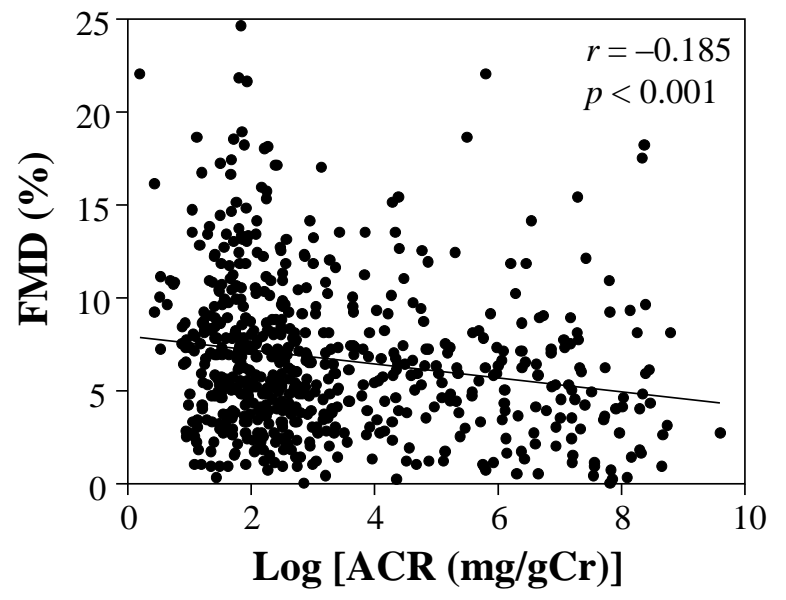

D

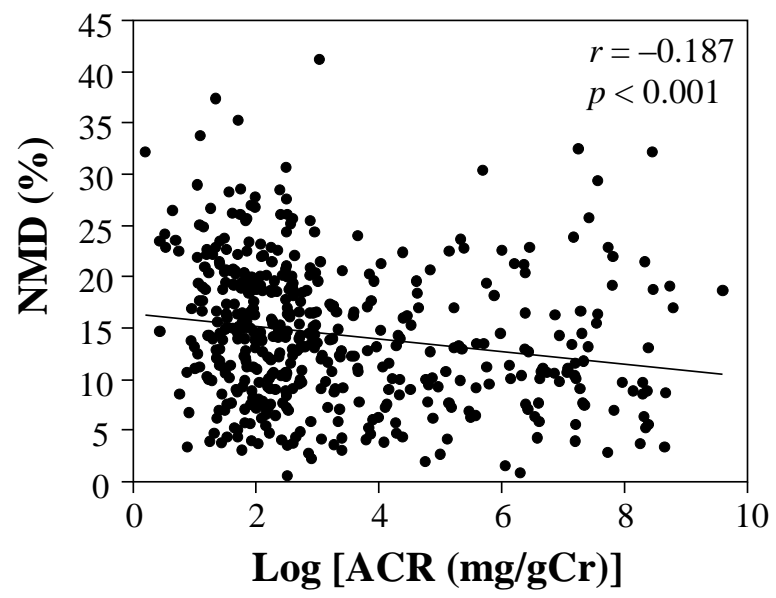


Table 1. Clinical characteristics and vascular endothelial function in all subjects

\begin{tabular}{|c|c|c|}
\hline & All subjects & Full-case population \\
\hline $\mathrm{N}$ (male/female) & $633(363 / 270)$ & $539(308 / 231)$ \\
\hline Age (year) & $62.0 \pm 12.7$ & $62.6 \pm 12.7$ \\
\hline Duration of diabetes (year) & $10(4-20)$ & $11(5-20)$ \\
\hline $\operatorname{BMI}\left(\mathrm{kg} / \mathrm{m}^{2}\right)$ & $26.0 \pm 5.5$ & $25.9 \pm 5.5$ \\
\hline Systolic blood pressure $(\mathrm{mmHg})$ & $131 \pm 20$ & $131 \pm 20$ \\
\hline Diastolic blood pressure (mmHg) & $74 \pm 10$ & $74 \pm 10$ \\
\hline Cardiovascular diseases $(n, \%)$ & $126(19.9)$ & $112(20.8)$ \\
\hline Coronary artery disease $(n, \%)$ & $62(9.8)$ & $53(9.9)$ \\
\hline Cerebrovascular disease $(n, \%)$ & $54(8.5)$ & $49(9.1)$ \\
\hline Peripheral artery disease $(n, \%)$ & $37(5.8)$ & $35(6.5)$ \\
\hline Statin $(n, \%)$ & $279(44.1)$ & $243(45.1)$ \\
\hline RAAS inhibitors $(n, \%)$ & $252(39.8)$ & $215(39.9)$ \\
\hline Smoker $(n, \%)$ & $307(48.5)$ & $254(47.1)$ \\
\hline Fasting glucose (mg/dL) & $125 \pm 36$ & $125 \pm 35$ \\
\hline HbA1c (\%) & $8.6 \pm 1.8$ & $8.7 \pm 1.8$ \\
\hline Serum creatinine $(\mathrm{mg} / \mathrm{dL})$ & $1.05 \pm 0.84$ & $1.04 \pm 0.79$ \\
\hline $\mathrm{eGFR}\left(\mathrm{mL} / \mathrm{min} / 1.73 \mathrm{~m}^{2}\right)$ & $66.5 \pm 27.4$ & $66.2 \pm 26.9$ \\
\hline Total cholesterol (mg/dL) & $181 \pm 48$ & $179 \pm 45$ \\
\hline Triglycerides (mg/dL) & $116(87-156)$ & $114(86-149)$ \\
\hline HDL-cholesterol (mg/dL) & $44 \pm 12$ & $44 \pm 12$ \\
\hline LDL-cholesterol (mg/dL) & $111 \pm 40$ & $110 \pm 40$ \\
\hline Urinary ACR (mg/g) & $12.5(6.1-78.2)$ & $12.4(6.2-64.7)$ \\
\hline FMD $(\%)$ & $6.7 \pm 4.2$ & $6.6 \pm 4.1$ \\
\hline $\operatorname{NMD}(\%)^{*}$ & $14.3 \pm 6.8$ & $14.3 \pm 6.8$ \\
\hline
\end{tabular}

Data are expressed as $n(\%)$, mean \pm standard deviation, or median (interquartile range). ${ }^{*}, n=$ 473 in all subjects and $n=394$ in full-case population after excluding those with contraindications to nitroglycerin. BMI, body mass index; RAAS, renin-angiotensin-aldosterone system; HbAlc, glycated hemoglobin Alc; eGFR, estimated glomerular filtration rate; HDL, high-density lipoprotein; LDL, low-density lipoprotein; ACR, albumin-to-creatinine ratio; FMD, flow-mediated dilatation; NMD, nitroglycerin-mediated dilatation. 
Table 2. Clinical characteristics of each chronic kidney disease stage based on eGFR or albuminuria

\begin{tabular}{|c|c|c|c|c|c|c|c|c|c|}
\hline & \multicolumn{5}{|c|}{ eGFR categories $\left(\mathrm{mL} / \mathrm{min} / 1.73 \mathrm{~m}^{2}\right)$} & \multicolumn{4}{|c|}{ Albuminuria categories } \\
\hline & $\begin{array}{l}\text { G1/2 } \\
(\geq 60)\end{array}$ & $\begin{array}{c}\text { G3a } \\
(45-59)\end{array}$ & $\begin{array}{c}\text { G3b } \\
(30-44)\end{array}$ & $\begin{array}{l}\text { G4/5 } \\
(<30)\end{array}$ & $p$ & $\begin{array}{c}\mathrm{A1} \\
(<30 \mathrm{mg} / \mathrm{g})\end{array}$ & $\begin{array}{c}\text { A2 } \\
(30-299 \mathrm{mg} / \mathrm{g})\end{array}$ & $\begin{array}{c}\mathrm{A3} \\
(\geq \mathbf{3 0 0} \mathrm{mg} / \mathrm{g})\end{array}$ & $p$ \\
\hline $\mathrm{N}$ (male/female) & $\begin{array}{c}392 \\
(215 / 177)\end{array}$ & $\begin{array}{c}118 \\
(73 / 45)\end{array}$ & $\begin{array}{c}63 \\
(39 / 24)\end{array}$ & $\begin{array}{c}60 \\
(36 / 24)\end{array}$ & 0.003 & $\begin{array}{c}425 \\
(231 / 194)\end{array}$ & $\begin{array}{c}101 \\
(61 / 40)\end{array}$ & $\begin{array}{c}107 \\
(71 / 36)\end{array}$ & 0.007 \\
\hline Age (year) & $59.0 \pm 12.9$ & $66.4 \pm 11.4^{*}$ & $67.5 \pm 10.3^{*}$ & $67.6 \pm 10.5^{*}$ & $<0.001$ & $62.3 \pm 12.7$ & $61.8 \pm 12.8$ & $62.3 \pm 13.1$ & 0.749 \\
\hline $\begin{array}{l}\text { Duration of diabetes } \\
\text { (year) }\end{array}$ & $10.8 \pm 9.9$ & $15.3 \pm 11.1^{*}$ & $16.7 \pm 10.7 *$ & $18.5 \pm 9.8^{*}$ & $<0.001$ & $11.7 \pm 10.4$ & $14.2 \pm 10.6$ & $16.9 \pm 10.1^{*}$ & $<0.001$ \\
\hline BMI $\left(\mathrm{kg} / \mathrm{m}^{2}\right)$ & $26.3 \pm 6.0$ & $25.5 \pm 4.6$ & $25.9 \pm 4.9$ & $25.3 \pm 4.5$ & 0.429 & $25.9 \pm 5.3$ & $26.5 \pm 6.6$ & $25.8 \pm 5.0$ & 0.642 \\
\hline $\begin{array}{l}\text { Systolic blood } \\
\text { pressure }(\mathrm{mmHg})\end{array}$ & $128 \pm 18$ & $134 \pm 18^{*}$ & $132 \pm 23$ & $146 \pm 25^{*}$ & $<0.001$ & $127 \pm 18$ & $132 \pm 20^{*}$ & $148 \pm 21^{*}$ & $<0.001$ \\
\hline Statin $(n, \%)$ & $146(38.3)$ & $67(57.8)$ & $34(54.0)$ & $32(55.2)$ & 0.001 & $176(42.2)$ & $45(45.5)$ & $58(56.9)$ & 0.029 \\
\hline $\begin{array}{l}\text { RAAS inhibitors } \\
(n, \%)\end{array}$ & 113 (29.7) & $56(48.7)$ & $38(60.3)$ & 45 (77.6) & $<0.001$ & $135(32.7)$ & $47(47.5)$ & $70(66.7)$ & $<0.001$ \\
\hline Smoker $(n, \%)$ & $188(48.1)$ & $58(49.2)$ & $29(46.0)$ & $32(53.3)$ & 0.859 & $200(47.1)$ & $48(48.0)$ & $59(55.1)$ & 0.324 \\
\hline $\begin{array}{l}\text { Cardiovascular } \\
\text { diseases }(n, \%)\end{array}$ & $49(12.5)$ & $31(26.3)$ & $20(32.8)$ & $26(43.3)$ & $<0.001$ & $71(16.8)$ & $24(23.8)$ & $31(29.3)$ & 0.010 \\
\hline $\begin{array}{l}\text { Fasting glucose } \\
(\mathrm{mg} / \mathrm{dL})\end{array}$ & $128 \pm 35$ & $122 \pm 34$ & $121 \pm 38$ & $123 \pm 39$ & 0.261 & $124 \pm 32$ & $131 \pm 46$ & $126 \pm 38$ & 0.165 \\
\hline HbAlc (\%) & $8.8 \pm 1.9$ & $8.5 \pm 1.5$ & $8.7 \pm 1.7$ & $7.7 \pm 1.8 *$ & 0.001 & $8.6 \pm 1.7$ & $9.0 \pm 1.9$ & $8.7 \pm 2.1$ & 0.079 \\
\hline $\begin{array}{l}\text { HDL-cholesterol } \\
(\mathrm{mg} / \mathrm{dL})\end{array}$ & $44 \pm 13$ & $44 \pm 12$ & $43 \pm 11$ & $39 \pm 9 *$ & 0.005 & $44 \pm 13$ & $43 \pm 12$ & $42 \pm 11$ & 0.261 \\
\hline $\begin{array}{l}\text { LDL-cholesterol } \\
(\mathrm{mg} / \mathrm{dL})\end{array}$ & $113 \pm 40$ & $107 \pm 42$ & $101 \pm 32$ & $115 \pm 44$ & 0.107 & $109 \pm 36$ & $111 \pm 35$ & $119 \pm 58$ & 0.102 \\
\hline
\end{tabular}

Data are expressed as $n(\%)$ or mean \pm standard deviation. $P$-values were determined by using ANOVA or $\chi^{2}$-test. *, $p<0.05$ by ANOVA with post-hoc Dunn's test as compared to G1/2 stage or A1 stage.

Abbreviations are as in Table 1. 
Table 3. Multiple regression analysis of the determinants for FMD or NMD in subjects with type 2 diabetes

\begin{tabular}{|c|c|c|c|c|c|c|}
\hline & \multicolumn{3}{|c|}{ FMD } & \multicolumn{3}{|c|}{ NMD } \\
\hline & model 1 & model 2 & $\overline{\text { model } 3}$ & model 1 & model 2 & model 3 \\
\hline Age & -0.080 & $-0.148 * *$ & $-0.130^{*}$ & $-0.245 * *$ & $-0.285^{* *}$ & $-0.266^{* *}$ \\
\hline Sex $($ male $=1$, female $=0)$ & $-0.135^{*}$ & $-0.132 * *$ & $-0.130 * *$ & $-0.124^{*}$ & $-0.126^{*}$ & $-0.123 *$ \\
\hline Duration of diabetes & 0.056 & 0.068 & 0.069 & -0.045 & -0.040 & -0.039 \\
\hline BMI & 0.019 & 0.009 & 0.009 & $-0.133^{*}$ & $-0.134^{*}$ & $-0.135^{*}$ \\
\hline Systolic blood pressure & -0.069 & -0.021 & -0.021 & $-0.114^{*}$ & -0.097 & -0.096 \\
\hline $\mathrm{HbA} 1 \mathrm{c}$ & -0.079 & -0.058 & -0.064 & -0.015 & -0.003 & -0.009 \\
\hline LDL-cholesterol & -0.004 & 0.001 & 0.004 & -0.019 & -0.019 & -0.015 \\
\hline Smoker $($ yes $=1$, no $=0)$ & 0.002 & 0.004 & 0.004 & 0.078 & 0.082 & 0.081 \\
\hline $\begin{array}{l}\text { Cardiovascular diseases } \\
(\mathrm{yes}=1, \mathrm{no}=0)\end{array}$ & $-0.117^{*}$ & $-0.122 * *$ & $-0.119^{*}$ & $-0.129^{*}$ & $-0.133 *$ & $-0.130^{*}$ \\
\hline Statin $($ yes $=1$, no $=0)$ & 0.010 & 0.019 & 0.020 & 0.026 & 0.032 & 0.033 \\
\hline $\begin{array}{l}\text { RAAS inhibitors (yes }=1 \text {, } \\
\text { no }=0 \text { ) }\end{array}$ & -0.084 & -0.067 & -0.065 & -0.011 & -0.008 & -0.003 \\
\hline Insulin $($ yes $=1$, no $=0)$ & -0.014 & -0.010 & -0.008 & -0.042 & -0.039 & -0.038 \\
\hline Sulfonylureas $($ yes $=1$, no $=0$ ) & $-0.094^{*}$ & -0.089 & -0.090 & 0.025 & 0.028 & 0.026 \\
\hline Biguanides $($ yes $=1$, no $=0$ ) & 0.026 & 0.021 & 0.019 & 0.012 & 0.011 & 0.010 \\
\hline $\begin{array}{l}\text { DPP-4 inhibitors (yes }=1 \text {, } \\
\text { no }=0 \text { ) }\end{array}$ & -0.080 & -0.077 & -0.077 & -0.066 & -0.064 & -0.065 \\
\hline eGFR & 0.101 & & 0.038 & 0.068 & & 0.042 \\
\hline $\log [\mathrm{ACR}]$ & & $-0.158 * *$ & $-0.143 * *$ & & -0.076 & -0.060 \\
\hline$\overline{R^{2}}$ & $0.101^{* *}$ & $0.113^{* *}$ & $0.113^{* *}$ & $0.168^{* *}$ & $0.170^{* *}$ & $0.170^{* *}$ \\
\hline
\end{tabular}

Values are standardized regression coefficients $(\beta)$ by multiple regression analysis. $R^{2}$, coefficient of determination. *, $p<0.05 ; * *, p<0.01$. DPP, dipeptidyl peptidase. The other abbreviations are as in Table 1. 